\title{
Editorial
}

\section{The nucleolus fine-tunes the orchestration of an early neuroprotection response in neurodegeneration}

\author{
JD Erickson ${ }^{\star, 1}$ and NG Bazan ${ }^{\star, 1}$ \\ Cell Death and Differentiation (2013) 20, 1435-1437; doi:10.1038/cdd.2013.107
}

The nucleolus is the cellular center of ribosome biogenesis necessary for neuronal survival. ${ }^{1,2}$ Impaired nucleolar activity-or nucleolar stress-may occur at early neurodegenerative disease stages and contribute to pathogenesis. ${ }^{3-5}$ In post-mortem specimens of human Huntington's disease (HD), insoluble aggregates of huntingtin-a hallmark of this disorder - are found in the nucleolus, ${ }^{6}$ which may be linked to impaired rDNA transcription, nucleolar dysfunction and specific degeneration of the striatum. An intriguing role of the nucleolus in cellular homeostasis is its participation in sensing cellular stress signals and transmitting them to the p53 stabilization system. ${ }^{7}$ In response to nucleolar stress, various nucleolar proteins are released to the nucleoplasm where they affect the activity of MDM2, a E3 ubiquitin ligase. Normally, this enzyme promotes proteasomal degradation of the transcription factor p53 but is impaired by the nucleolar proteins, leading to accumulation of p53. Post-translational modifications of p53 promote apoptosis, which has important implications in neurodegenerative disorders. ${ }^{8,9}$ While stabilization and activation of p53 occurs in response to an assorted array of cellular stress ranging from hypoxia, excitotoxicity, to intracellular expression of misfolded proteins, an intriguing view is that a common site for these varied cellular insults to activating p53 is damage to the nucleolus.

Another p53-regulated cellular process is autophagy, a complex lysosomal pathway of cellular self-digestion, which represents a mechanism used to adapt to different forms of cellular stresses. ${ }^{10}$ Autophagy is responsible for the degradation of normal proteins involved in cell remodeling as well as for the digestion and removal of abnormal proteins and damaged organelles which accumulate in various neurodegenerative disorders. ${ }^{11,12}$ An important question is whether autophagy is a survival mechanism and to establish whether autophagy is genetically upstream of various death pathways, such as apoptosis or necrosis. ${ }^{13}$ The role of autophagy in protection against neurodegenerative diseases has been established in animal models, ${ }^{14}$ and while autophagy is present in $\mathrm{HD}$ and $\mathrm{AD}$ brain, ${ }^{15}$ neurons in the final stages of their death often display counteracting pro-death factors and pro-survival responses. In this issue, results in the study by Kreiner and co-workers ${ }^{16}$ provide further evidence that autophagy has a protective role in the early stages of neurodegeneration ${ }^{16-18}$ using a model of HD as part of a desperate attempt by the cell to save itself from destruction (see Figure 1).
The model utilized is based on the genetic ablation of TIF-1A, an evolutionary conserved transcription factor essential for the recruitment of RNA polymerase I to rRNA promoters. Targeted ablation of TIF-IA in medium spiny neurons (MSNs) using dopamine receptor (D1R) transgenic mice (TIF-IA ${ }^{\text {D1RCre }}$ ) causes inhibition of pre-rRNA synthesis, loss of mature rRNA, nucleolar structure disruption, accumulation of p53, and progressive degeneration of the striatum and HD-like abnormalities. The increase in p53 in MSNs in TIF-IA ${ }^{\text {D1RCre }}$ mice included acetylation at LYS373 and Lys382, which is thought to be required for p53-dependent induction of apoptosis. Intriguingly, the loss of p53 does not ameliorate the effects of the loss of TIF-IA and subsequent nucleolar stress on neuronal survival or motor impairment. Inactivation of the TIF-IA gene in adult dopaminergic (DA) neurons by a druginducible approach in adult mice leads to a phenotype closely resembling Parkinson's disease (PD). ${ }^{4}$ At the mechanistic level, this nucleolar stress resulted in similar increased p53 levels and downregulation of mammalian target of rapamycin (mTOR) activity. ${ }^{4}$ However, inhibition of p53 pharmacologically (pifithrin- $\alpha$ treatment) or genetically in TIF-IA;p53double mutants blocked the loss of DA neurons, illustrating the importance of p53 for the PD-like pathogenesis. ${ }^{4}$ These divergent results indicate possible context-specific roles of nucleolar-dependent functions and p53 in neuronal subtypes. Alternatively, the disparate roles of p53 in these two animal models may reveal different time courses for neurodegeneration, which in humans progresses over many years.

Gene expression profiles indicated that PTEN (phosphatase and tensin homolog deleted on chromosome 10) was upregulated MSN's in TIF-IA ${ }^{\text {D1RCre }}$ mice after 9 weeks, but not after 13 weeks, when apoptosis was prominent. The authors suggest that PTEN suppresses the phosphatidylinositol3-kinase (PI3K/AKT/mTOR) pathway as phosphorylation of the ribosomal protein S6, a downstream target of mTOR, was decreased in 9-week-old TIF-IA ${ }^{\text {D1RCre }}$ mice. Similarly, phosphorylation of the eukaryotic initiation factor $4 \mathrm{E}$ binding protein, a direct $\mathrm{mTOR}$ target, was reduced in TIF-IA ${ }^{\mathrm{D} 1 \mathrm{RCre}}$ mice. The authors generated TIF-IA;PTEN ${ }^{\text {D1RCre }}$-double mutant mice and $\mathrm{S} 6$ phosphorylation was not reduced in the striata. Consistent with PTEN being regulated by $\mathrm{p} 53$ expression, PTEN was not increased in striata of TIF-IA;p53 ${ }^{\text {D1RCre }}$ double mutant mice. These data support the notion that p53-dependent upregulation of PTEN impairs mTOR function

\footnotetext{
${ }^{1}$ Neuroscience Center of Excellence, School of Medicine, Louisiana State University Health Sciences Center, New Orleans, LA, USA

${ }^{*}$ Corresponding author: NG Bazan and JD Erickson, Neuroscience Center of Excellence, School of Medicine, Louisiana State University, Health Sciences Center, New Orleans, LA 70112, USA. Tel: 504599 0831; Fax: 504568 5801; E-mail: nbazan@Isuhsc.edu and jerick@Isuhsc.edu
} 


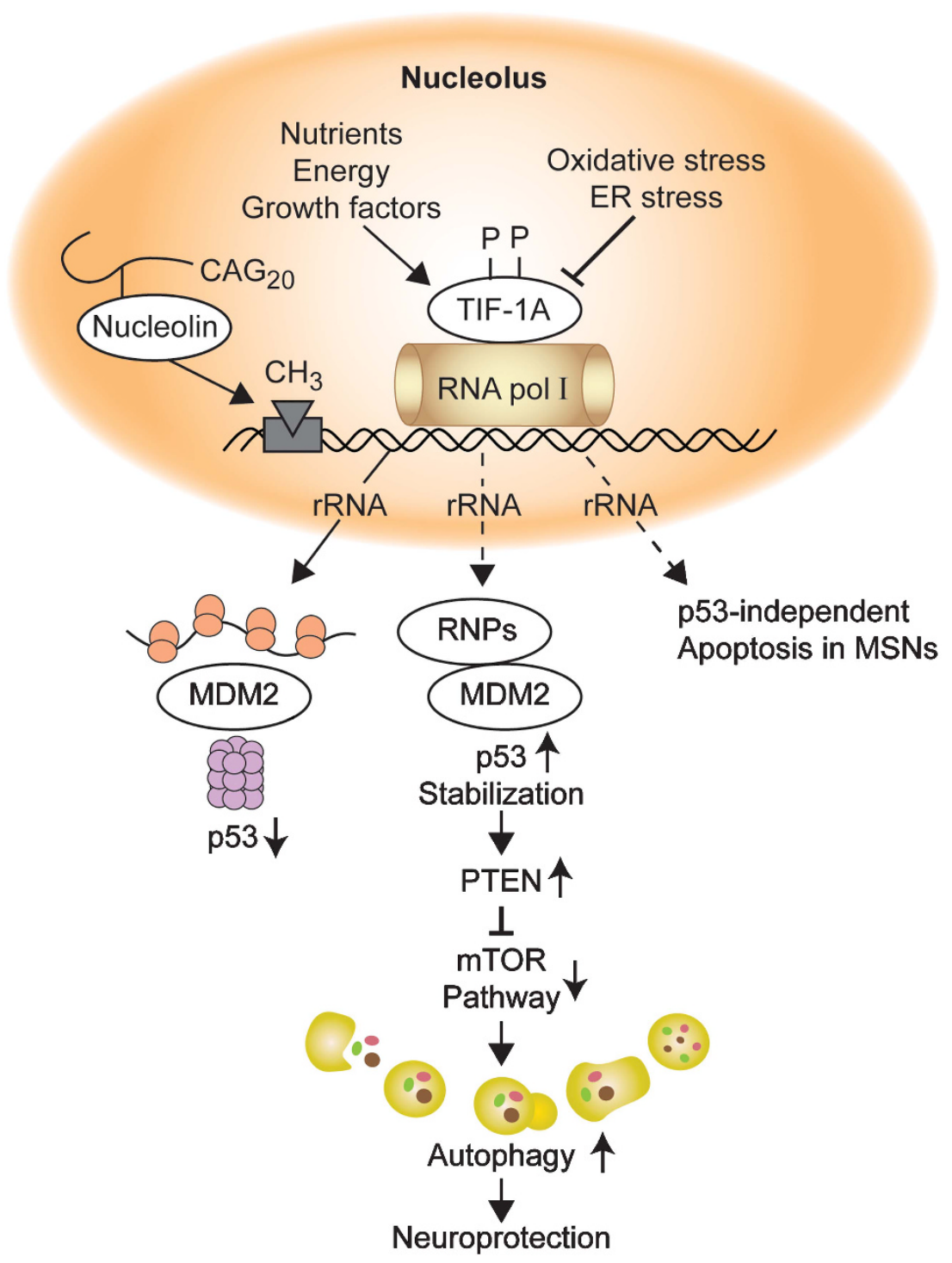

Figure 1 Hierarchy of nucleolar stress-induced neuroprotection. RNA polymerase I (RNA pol I) is critical for the synthesis of ribosomal RNA (rRNA) and ribosomes, and is regulated by the transcription factor TIF-IA. A variety of protein kinases activated in response to mitogenic signals, growth stimuli, and cellular energy status or in response to oxidative or endoplasmic reticulum stress regulate TIF-IA at distinct serine residues. In HD, the action of nucleolin, which is required for RNA pol I-mediated transcription, is interfered with by expanded CAG RNA molecules and leads to $\mathrm{CpG}$ hypermethylation of upstream control elements in the RNA pol I promoter and a reduction in rRNA transcription. Under normal conditions the E3 ubiquitin ligase MDM2 (in mice; HDM2 in human) and proteasome-dependent degradation keeps the transcription factor p53 at low basal levels. Following nucleolar stress various nucleolar proteins (RNPs) inactivate MDM2, thereby stabilizing and increasing p53 levels. Elevated nuclear p53 levels lead to upregulation of phosphatase and tensin homolog deleted on chromosome 10 (PTEN), which suppresses the mTOR pathway, which is the major inhibitory signal that shuts off autophagy in the presence of growth factors and abundant nutrients. While p53-dependent induction of PTEN, inhibition of mTOR signaling, and autophagy promote neuronal survival and delay neuronal death, MSNs eventually still succumb to p53-independent apoptosis

in MSNs. mTOR signaling is a major inhibitory signal that shuts off autophagy. ${ }^{10}$ Electron microscopy evidence in TIF-IA ${ }^{\text {DIRCre }}$ mice revealed the formation of autophagosomes in striatum at 9 weeks. Abrogation of TIF-IA in combination with p53 or PTEN prevents the decrease of autophagy-related proteins LC3-I and p62 at 9 weeks. In addition, increased acetylation of p53 and apoptosis was sparse at 9 weeks but significant at 13 weeks. Consistent with autophagy being a means to prolong survival, the amount of apoptotic cells in MSNs from 9-week-old TIF-IA;PTEN ${ }^{\text {D1RCre }}$ and TIF-IA;p53 ${ }^{\text {D1RCre }}$ mice was significantly increased. This suggests that macroautophagy preceded apoptotic cell death and that upregulation of $\mathrm{p} 53$ in TIF-IA ${ }^{\text {DIRCre }}$ mice leads to a transient pro-survival response: $p 53-d e p e n d e n t$ induction of PTEN, inhibition of mTOR signaling, and autophagy.

The Kreiner et al. ${ }^{16}$ study highlights that TIF-IA mutant mice can be used to advance and authenticate therapeutic intervention to promote autophagy as a survival mechanism and discover biomarkers associated with this degenerative model. The cellular decisions between autophagy or apoptotic death pathways include potentially variable thresholds for each process, the role of differential post-translational processing of $\mathrm{p} 38$, and mutual inhibition or activation of each pathway by the other. ${ }^{13}$ To acquire a better understanding of 
the signaling control of autophagy and to develop specific ways to stimulate autophagy, further study of the mechanistic basis for age-related, cellular context- and disease-specific defects in autophagy activation is required. P53-dependent induction of PTEN, inhibition of mTOR signaling, and

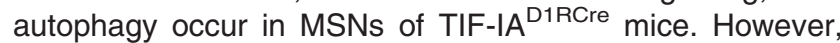
inhibition of PTEN-dependent functions in adult DA neurons activates mTOR signaling and may represent a promising PD therapy. ${ }^{19}$ Autophagy may reach a point of saturation, and its capacity to degrade the mutant aggregate-prone proteins is exceeded. In a transgenetic HD mouse model, autophagyenhancing drugs decreased aggregate formation and improved HD-associated behavioral tasks. ${ }^{12}$ Nuclear versus cytoplasmic p53 expression is regulated by discrete signaltransduction pathways to mediate neuronal survival and apoptotic cell death. ${ }^{9,10}$ Further knowledge of key elements that define p53-mediated autophagy versus apoptosis in the stress response is a critical issue. Future investigation should also include the role of small non-coding RNAs regulating mRNA translation in the nucleolus ${ }^{20}$ and the regulation of microRNA processing and location as an additional mechanism linking nucleolar activity to protein synthesis regulation, ${ }^{21}$ which may lead to a functional link between the nucleolus and non-coding RNAs in neuronal survival. Although the studies of TIF-IA ablation in discrete neuronal populations do not prove that the nucleolar damage is a primary cause of neurodegeneration, it suggests that the nucleolus fine-tunes the orchestration of an early neuroprotective response.

\section{Conflict of Interest}

The authors declare no conflict of interest.

1. Hetman M, Pietrzak M. Trends Neurosci 2012; 35: 305-314.

2. Parlato R, Kreiner G. J Mol Med 2013; 91: 541-547.

3. Pietrzak $\mathrm{M}$ et al. PLoS One 2011; 6: e2585.

4. Rieker $C$ et al. J Neurosci 2011; 31: 453-460

5. Tsoi H et al. Proc Natl Acad Sci 2012; 109: 13428-13433.

6. Latonen L. Bioessays 2011; 33: 386-395

7. Boulon S et al. Mol Cell 2010; 40: 216-227.

8. Vousden KH, Lane DP. Nat Rev Mol Cell Biol 2007; 8: 275-283.

9. Chang JR et al. Neurodegener Dis 2012; 9: 68-80.

10. Tasdemir E et al. Nat Cell Biol 2008; 10: 676-687.

11. Kegel KB et al. J Neurosci 2000; 20: 7268-7278.

12. Gorman AM. J Cell Mol Med 2008; 12: 2263-2280.

13. Maiuri MC et al. Nat Rev Mol Cell Biol 2007; 8: 741-752.

14. Mizushima N et al. Nature 2008; 451: 1069-1075.

15. Rubinsztein DC et al. Nat Rev Drug Discov 2007; 6: 304-312.

16. Kreiner $\mathrm{G}$ et al. Cell Death Differ 2013; 20: 1455-1464.

17. Hara T et al. Nature 2006; 441: 885-889.

18. Komatsu $M$ et al. Nature 2006; 441: $880-884$

19. Domanskyi A et al. FASEB J 2011; 25: 2898-2910.

20. Politz JC, Hogan EM, Pederson T. RNA 2009; 15: 1705-1715.

21. Janas MM et al. Mol Cell 2012; 46: 171-186. 